\title{
UK Renal Registry 16th Annual Report: Appendix K Renal Centre Names and Abbreviations used in the Figures and Data Tables
}

\section{Adult Centres}

\section{City}

England

Basildon

Birmingham

Birmingham

Bradford

Brighton

Bristol

Cambridge

Carlisle

Carshalton

Chelmsford

Colchester

Coventry

Derby

Doncaster

Dorset

Dudley

Exeter

Gloucester

Hull

Ipswich

Kent

Leeds

Leicester

Liverpool

Liverpool

London

London

London

London

London

London

Manchester

Middlesbrough

Newcastle

Norwich
Hospital

Basildon Hospital

Heartlands Hospital

Queen Elizabeth Hospital

St Luke's Hospital

Royal Sussex County Hospital

Southmead Hospital

Addenbrooke's Hospital

Cumberland Infirmary

St Helier Hospital

Broomfield Hospital

Colchester General Hospital

University Hospital Coventry

Royal Derby Hospital

Doncaster Royal Infirmary

Dorset County Hospital

Russells Hall Hospital

Royal Devon and Exeter Hospital

Gloucestershire Royal Hospital

Hull Royal Infirmary

Ipswich Hospital

Kent and Canterbury Hospital

St James's University Hospital and Leeds General Infirmary

Leicester General Hospital

Aintree University Hospital

Royal Liverpool University Hospital

St. Bartholomew's Hospital and The Royal London Hospital

St George's Hospital and Queen Mary's Hospital

Guy's Hospital and St Thomas' Hospital

Hammersmith, Charing Cross, St Mary's

King's College Hospital

Royal Free, Middlesex and UCL Hospitals

Manchester Royal Infirmary

The James Cook University Hospital

Freeman Hospital and Royal Victoria Infirmary

Norfolk and Norwich University Hospital
Abbreviation

Basldn

B Heart

B QEH

Bradfd

Brightn

Bristol

Camb

Carlis

Carsh

Chelms

Colchr

Covnt

Derby

Donc

Dorset

Dudley

Exeter

Glouc

Hull

Ipswi

Kent

Leeds

Leic

Liv Ain

Liv RI

L Barts

L St. G

L Guys

L West

L Kings

L Rfree

M RI

Middlbr

Newc

Norwch

\section{KARGER}

Fax +41 613061234

E-Mail karger@karger.com

www.karger.com
UK Renal Registry, Southmead Hospital, Southmead Road, Bristol, BS10 5NB, UK

Email: renalregistry@renalregistry.nhs.uk 


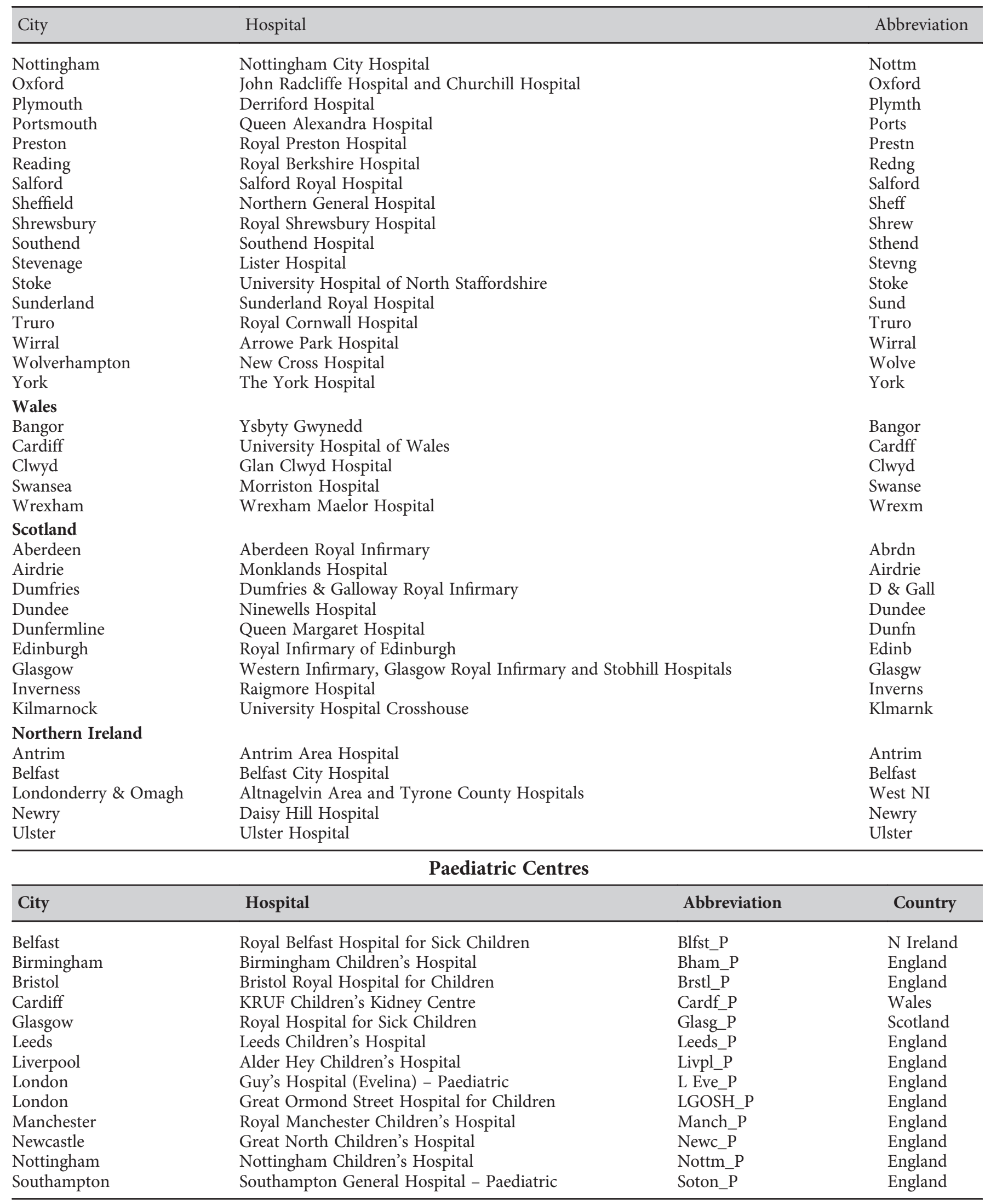

